\title{
"Click" Dendrimers as Anti-inflammatory Agents: With Insights into Their Binding from Molecular Modeling Studies
}

Kevin Neibert, ${ }^{\dagger}$ Varin Gosein, ${ }^{\dagger}$ Anjali Sharma, ${ }^{\ddagger}$ Mohidus Khan,${ }^{\ddagger}$ Michael A. Whitehead, ${ }^{*}$, Dusica Maysinger, ${ }^{*},{ }^{\dagger}$ and Ashok Kakkar, ${ }^{*}$

${ }^{\dagger}$ Department of Pharmacology and Therapeutics, McGill University 3655 Promenade Sir-William-Osler, Montreal, H3G 1Y5, Canada

${ }^{\ddagger}$ Department of Chemistry, McGill University, 801 Sherbrooke St. West, Montreal, Quebec, H3A 2K6, Canada

\section{Supporting Information}
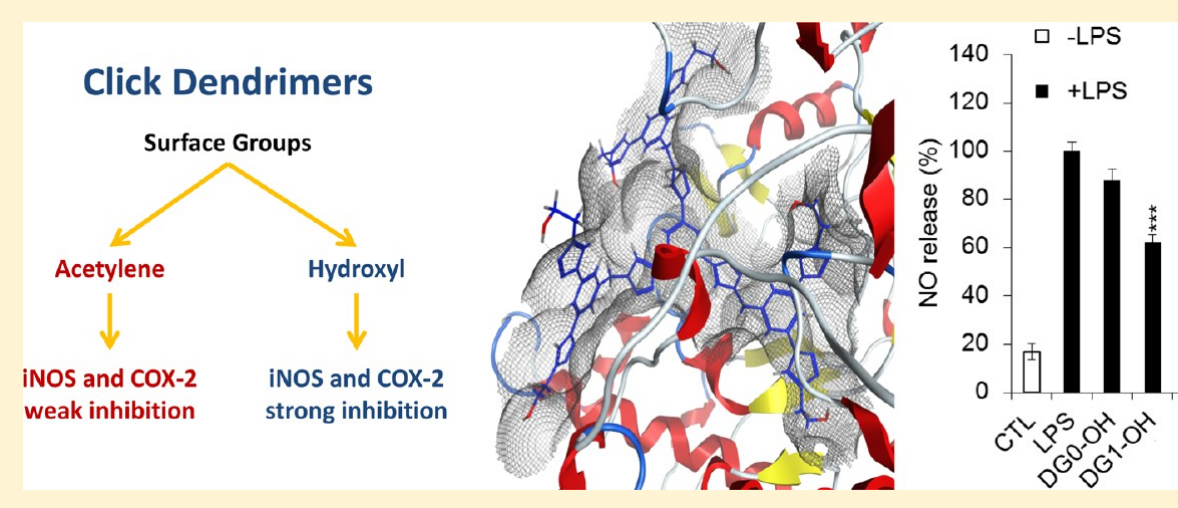

ABSTRACT: These studies explore the relationship between the inhibitory actions of low generation dendrimers in stimulated microglia and dendrimer-enzyme interactions using in silico molecular modeling. Low generation (DG0 and DG1) dendrimers with acetylene and hydroxyl terminal groups were tested for their anti-inflammatory activity in microglia stimulated by lipopolysaccharides (LPS), and the results were compared with those from the established anti-inflammatory agents, ibuprofen and celecoxib. We hypothesized that hydroxyl terminal groups of DG0 and DG1 dendrimers could interact with the active sites of the inducible nitric oxide synthase (iNOS) and cyclooxygenase-2 (COX-2) enzymes due to their small size and favorable electrochemical properties. The enzymatic activity of iNOS and COX-2 was determined in the presence of low generation dendrimers using biochemical assays and their values related to dendrimer docking confirmations from in silico molecular modeling. We found that results from the molecular modeling studies correlated well with the in vitro biological data, suggesting that, indeed, hydroxyl terminal groups of low generation dendrimers enable multivalent macromolecular interactions, resulting in the inhibition of both iNOS and COX-2 enzymes.

KEYWORDS: anti-inflammatory, dendrimers, microglia, molecular modeling, iNOS, COX

\section{INTRODUCTION}

Dendrimers have been extensively investigated as nanocarriers for therapeutic delivery including anti-inflammatory agents. ${ }^{1,2}$ The present paradigm for anti-inflammatory therapy involves the inhibition of the arachidonic acid pathway and prostaglandin synthesis by means of targeting the cyclooxygenase enzymes (COX-1 and COX-2). Due to the persistence of adverse side effects, particularly irritation and ulceration of the gastric mucosa by COX-1 inhibitors, therapeutic strategies that specifically target COX-2 have been explored. Nonsteroidal anti-inflammatory drugs (NSAIDs) that target COX-2 are effective anti-inflammatory agents and lack serious adverse effects on the gastro-duodenum. ${ }^{3}$ However, highly selective COX-2 inhibitors (e.g., celecoxib) can produce cardiovascular complications; therefore the search for new antiinflammatory agents is still an active area of research. ${ }^{4}$ One such strategy involves the targeting of inducible nitric oxide synthase (iNOS), the primary cellular producer of nitric oxide, which is known to modulate inflammatory events. ${ }^{5}$ It is anticipated that the side effects arising from the inhibition of inducible proinflammatory enzymes (iNOS and COX-2) can be mitigated by specifically targeting therapeutics to inflamed tissues and by the controlled release from drug carriers or pro-drugs. Indeed, incorporation of anti-inflammatory agents into nanoscale delivery systems such as liposomes or polymers has been reported to significantly reduce adverse effects. ${ }^{6}$ Among them, dendrimers (hyperbranched polymers) have been thoroughly investigated as versatile drug delivery carriers, a function especially favored by their unique physical and chemical properties. ${ }^{7}$

Received: January 30, 2013

Revised: April 2, 2013

Accepted: April 16, 2013

Published: April 16, 2013 
Scheme 1. Synthesis of DG0 and DG1 Dendrimers with Acetylene (A) and Hydroxyl $(\mathrm{OH})$ Surface $\mathrm{Groups}(\mathrm{i}) \mathrm{CuSO}_{4} \cdot 5 \mathrm{H}_{2} \mathrm{O}$, Sodium Ascorbate, THF/ $\mathrm{H}_{2} \mathrm{O}$; (ii) $\mathrm{Bu}_{4} \mathrm{NF}$, THF; (iii) $\mathrm{NaN}_{3} / \mathrm{DMF}$

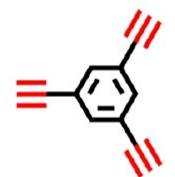

DG0-A

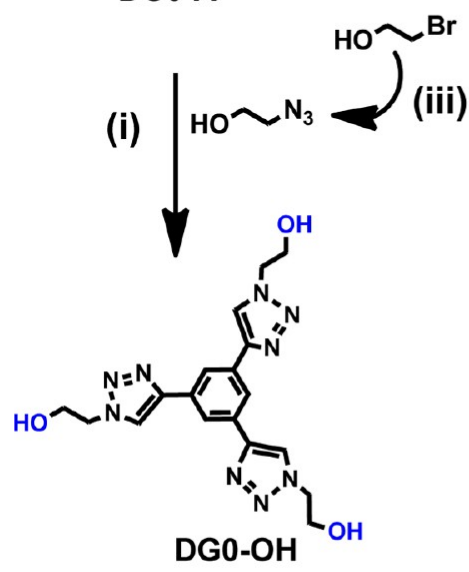

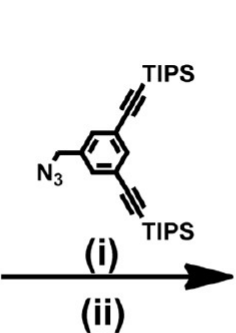

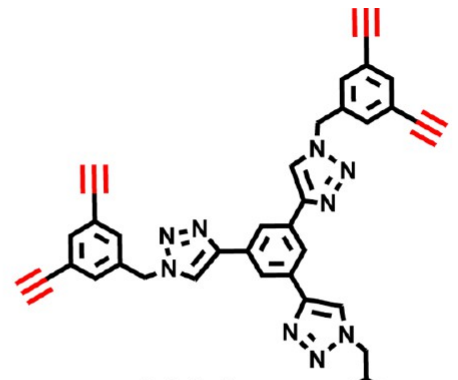

DG1-A

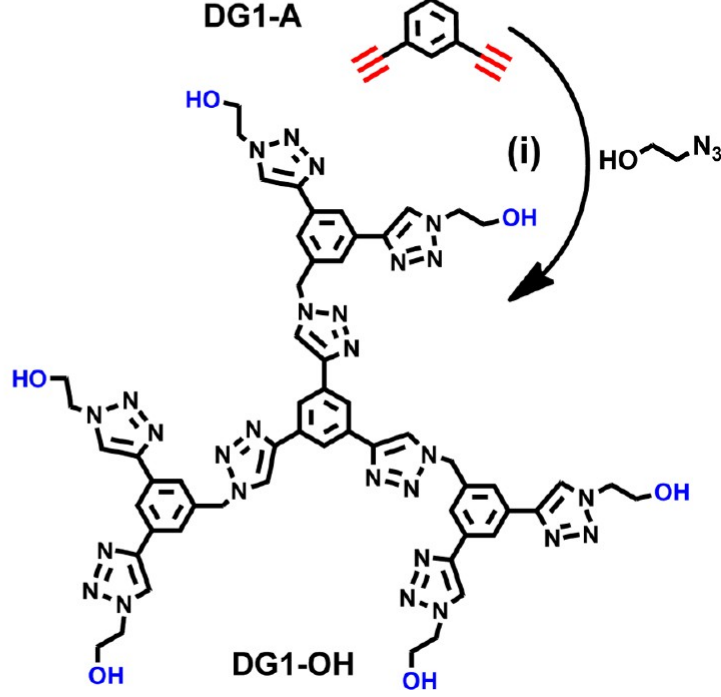

Poly(amidoamine) (PAMAM) dendrimers have been used for the controlled delivery of NSAIDs and steroidal antiinflammatory drugs. ${ }^{8,9}$ Recent studies suggest that dendrimer size plays a very important role in their efficiency as drug carriers. $^{10-12}$ Surprisingly, PAMAM dendrimers without any drug payload have also been shown to exhibit antiinflammatory properties, particularly generation 4 (G4) PAMAM dendrimers. ${ }^{12}$ It was suggested that the inhibition of COX-2 activity may explain some of the anti-inflammatory effects, although the specific mechanism(s) of G4 PAMAM action remain unclear. ${ }^{12}$ In addition, G3 dendritic polyglycerol sulfates (dPGS) also possess anti-inflammatory properties, though through a completely different mechanism of action. ${ }^{13}$ It is believed that dPGS dendrimers may function as macromolecular inhibitors, through direct interactions with complement factors, essential mediators of the innate immune response. $^{13}$

During our continued studies and development of dendrimer based multifunctional nanomaterials using alkyne-azide "click" chemistry, we too were intrigued to note that some of the nanocarriers were themselves active anti-inflammatory agents. ${ }^{14-16}$ Our nanoconstructs are smaller than the previously discussed PAMAM and dPGS dendrimers, leading us to investigate how these lower generation dendrimers might also exert anti-inflammatory properties. The aims of this study were to (i) establish a structure/anti-inflammatory activity relationship and (ii) better understand the mechanism of action by evaluating the effect of dendrimer size and surface functionalization on the inhibition of inducible inflammatory enzymes.

Dendrimers were synthesized using the highly efficient "click" chemistry approach (Scheme 1) to give uniformity and reproducibility in high yield reactions. Acetylene (A) terminated dendrimers DG0-A, DG1-A, and DG2-A were synthesized using an adaptation of our previously published procedure. ${ }^{17}$ Hydroxyl (OH) terminated DG0-OH was prepared by using copper(I) catalyzed alkyne azide click reaction between the acetylene arms of DG0-A and 2-azidoethanol, which in turn was synthesized from 2-bromo-ethanol upon reaction with sodium azide. Similarly, the synthesis of DG1-OH was achieved by performing a click reaction between alkyne arms of DG1-A and 2-azido-ethanol. The click reactions are easily monitored by ${ }^{1} \mathrm{H} \mathrm{NMR}$ in which the disappearance of alkyne resonance is accompanied by the appearance of triazole proton peaks. Detailed synthesis and characterization details of these dendrimers are provided as Supporting Information.

Enzymatic activities of iNOS and COX-2 were measured using established biochemical assays following activation with bacterial endotoxin lipopolysaccharides (LPS). LPS is a commonly used inflammagen which stimulates inflammatory cells, including microglia. ${ }^{18}$ Following LPS treatment, iNOS and COX-2 are inducibly expressed and activated in microglia cells, resulting in increased nitric oxide and prostaglandin production. ${ }^{19}$ We determined the extent of LPS-induced iNOS and COX-2 activation by measuring production of both the stable nitric oxide derivative nitrite $\left(\mathrm{NO}^{2-}\right)$ and prostaglandin E2 (PGE2), respectively. We then performed molecular modeling studies to provide a structural basis for the observed inhibition of iNOS and COX-2 by low generation dendrimers (DG0-DG1) functionalized with alkyne and hydroxyl surface groups. 


\section{EXPERIMENTAL SECTION}

Synthesis and Characterization of Dendrimers. DG0A, DG1-A and DG2-A were synthesized using our previously published procedures. ${ }^{14,17}$ DG2-OH can be similarly prepared from DG2-A upon reaction with 2-azido-ethanol. Azido ethanol was synthesized using a previously described procedure. ${ }^{15}$ Copper(II) sulfatepentahydrate $\left(\mathrm{CuSO}_{4} .5 \mathrm{H}_{2} \mathrm{O}\right)$ (>98.0\%), sodium ascorbate (NaAsc) (crystalline, 98\%), sodium azide $\left(\mathrm{NaN}_{3}\right)(>99.5 \%)$, tetrabutylammonium fluoride $\left(\mathrm{Bu}_{4} \mathrm{NF}\right)(1.0$ $\mathrm{M}$ in THF), and 2-bromo-ethanol (95\%) were purchased from Sigma-Aldrich Canada and used as received.

Cell Culture. Murine microglia (N9) cells obtained from Dr. Seguela (Montreal Neurological Institute, Montreal) were seeded in Iscove's modified Dulbecco's medium (IMDM) (12440, Gibco) containing 5\% of fetal bovine serum (FBS; 26140, Gibco) and 1\% penicillin-streptomycin (15140, Gibco). Cells were maintained at $37{ }^{\circ} \mathrm{C}, 5 \% \mathrm{CO}_{2}$ in a humidified atmosphere and were grown in serum containing media for $24 \mathrm{~h}$ before cell treatments to attain confluency. Cells were used between 10 and 30 passages. The media were aspirated, and cells were treated in serum-free media containing or dendrimers anti-inflammatory agents (ibuprofen and celecoxib) at the indicated concentrations for $24 \mathrm{~h}$. Serumfree media is used for the cell treatments to limit the potential interactions between dendrimers and proteins present within the serum containing media. Microglial cells were then activated with LPS (62326, Sigma; $100 \mathrm{ng} / \mathrm{mL}, 24 \mathrm{~h}$ ) in serum containing media ( $1 \% \mathrm{FBS})$ to maximize activation. Samples were collected for biochemical assays for mitochondrial metabolic activity, nitric oxide generation, and prostaglandin E2 synthesis, after the removal of the media containing drugs and washing with phosphate-buffered saline (PBS). All measurements were done in triplicate, and the experiments were performed at least three times.

Mitochondrial Metabolic Activity Determination by MTT Assay. Cells were seeded in 24-well plates (3526, Costar). Following treatment, 3-(4,5-dimethylthiazol-2-yl)-2,5diphenyltetrazolium bromide (MTT) solution $(0.5 \mathrm{mg} / \mathrm{mL}$; M2128, Sigma) was added to each well and incubated for 30 min at $37{ }^{\circ} \mathrm{C}$. The formed formazan crystals were dissolved in $500 \mu \mathrm{L}$ of dimethyl sulfoxide (DMSO; 154938, Sigma) and transferred to a 96-well plate to measure the absorbance (595 $\mathrm{nm}$, Benchmark microplate reader Bio-Rad, Canada). All absorbance values were within the linear range, and the comparisons between different treatments were made by converting the absorbance into percent changes relative to the vehicle-treated controls taken as $100 \%$.

Nitric Oxide Release. Cells were seeded in 24-well culture plates (3526, Costar). Following the treatments, cells were activated with LPS $(100 \mathrm{ng} / \mathrm{mL})$ for $24 \mathrm{~h}$ in $1 \%$ FBS-containing media. Supernatants $(50 \mu \mathrm{L})$ were collected and incubated with the Griess reagent (Sigma, G-4410; $50 \mu \mathrm{L}$ ) for $15 \mathrm{~min}$, after which the absorbance at $540 \mathrm{~nm}$ was measured with a microplate reader (Asys UVM 340).

COX-2 Activity. Cells were seeded in 24-well plates (3526, Costar). Following treatment with either with anti-inflammatory drugs (ibuprofen and celecoxib) or with dendrimers for 24 $\mathrm{h}$, microglia cells were activated using LPS (100 ng/mL; $24 \mathrm{~h}$ ), and prostaglandin E2 (PGE2) was measured using an enzymelinked immunosorbent assay (ELISA; Cayman, 514010) following the manufacturer protocols. The values for optical density measurements were obtained using a microplate reader (Asys UVM 340).

Limulus Amebocyte Lysate (LAL) Endotoxic Test. We performed the LAL assay as specified by Thermo Scientific Assay Kit (CAT No. 88282).

Statistics. Results are presented as mean \pm standard error of the mean (SEM). Data were analyzed by one-way analysis of variance (ANOVA) with the posthoc Dunnett's test. Statistically significant differences are indicated by $p^{*}<0.05, p^{* *}<$ $0.01, p^{* * *}<0.001$

Molecular Modeling Studies. A detailed description of the molecular modeling and parameters used is available in the Supporting Information. Briefly, putative dendrimer binding pockets from the iNOS and COX-2 crystal structures were identified and defined as prospective binding pockets for subsequent docking simulations (Supplemental Figures 2-3). Energy-minimized dendrimer structures were docked to the predicted binding pockets using the MOE docking module. The top three binding conformations with the lowest binding energy were then energy minimized into the enzyme binding site to obtain a relaxed docking solution. Finally, these three optimized conformations were validated by evaluating the (i) van Der Waals forces (VdW), (ii) electrostatic interactions, and (iii) lipophilic interactions between the predicted dendrimer binding conformations and the corresponding enzyme binding sites.

\section{RESULTS AND DISCUSSION}

The anti-inflammatory activity of low generation dendrimers with acetylene and hydroxyl terminal groups was evaluated in N9 murine microglia. These cells were selected because they are well-characterized and show a number of properties similar to those of human microglia. ${ }^{18}$ Microglia, the resident immune cells of the central nervous system (CNS), survey the microenvironment for the presence of damaged neurons, particulate matter and infectious agents. Upon activation by pro-inflammatory stimuli, such as LPS, microglia release several inflammatory mediators, including nitric oxide and cytokines.

We established the concentration-dependent effects of selected anti-inflammatory drugs in LPS-activated microglia (Figure 1). The effectiveness of the dendrimers and the selected anti-inflammatory agents was first assessed by measuring nitric oxide production (an indicator of microglia activation) in untreated and LPS-stimulated microglia. There was a marked reduction in nitric oxide release following dendrimer treatment (25-50 $\mu \mathrm{M}$; Figure 1A). Conversely, there was no significant reduction of nitric oxide release following the treatments with ibuprofen and celecoxib, also within the $25-50 \mu \mathrm{M}$ concentration range. Results showing the comparative effectiveness of low generation dendrimers (25 $\mu \mathrm{M})$ on nitric oxide release revealed that only DG1-OH can significantly reduce nitric oxide release, suggesting a potential inhibitory effect on iNOS. Interestingly, the treatments with DG0-OH and DG1-OH also led to the inhibition of prostaglandin (PGE2) synthesis, a measure of inducible COX-2 activity in activated microglia (Figure 1B). Significant COX-2 inhibition was achieved only with Celecoxib in the lower concentration $(25 \mu \mathrm{M})$ but not with ibuprofen. These results suggest that the mechanism of action of DG0-OH and $\mathrm{DG} 1-\mathrm{OH}$ dendrimers is concentration-dependent and may involve an interaction with iNOS and COX-2 in microglia. There was no significant decrease in cell viability or mitochondrial metabolic activity $(<50 \mu \mathrm{M})$ for all the 

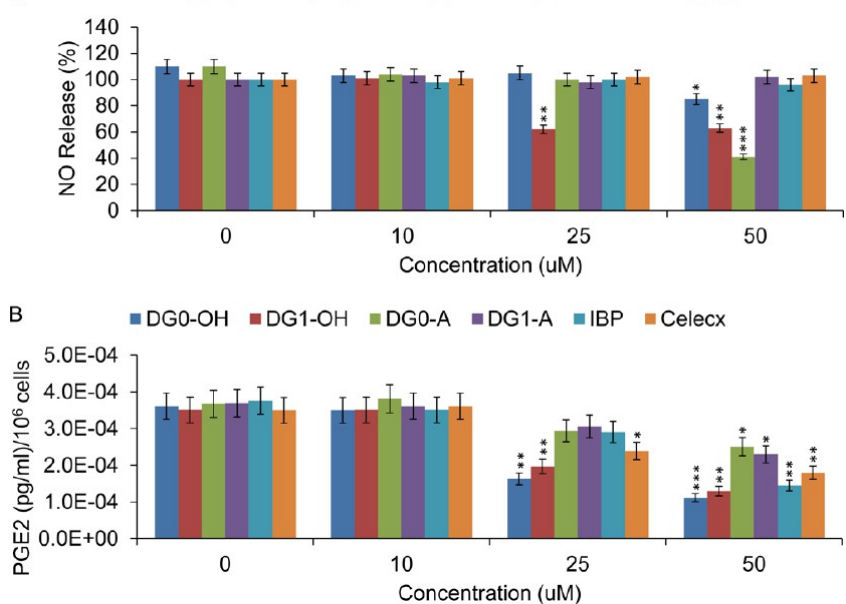

Figure 1. Concentration-dependent anti-inflammatory activity of dendeimrs. Reduction of (A) nitric oxide release and (B) prostaglandin E2 (PGE2) production by microglia, treated with dendrimers or anti-inflammatory agents $(0-100 \mu \mathrm{M}, 24 \mathrm{~h})$ in the presence of LPS (100 ng/mL, $24 \mathrm{~h}$ ). Data represent the mean \pm SEM. Statistically significant differences from LPS treated control are indicated by $p^{*}<0.05, p^{* *}<0.01, p^{* * *}<0.001$.

compounds tested (Supplemental Figure 1A). In addition, the dendrimers were found to be free of endotoxicity, measured by the LAL assay (Supplemental Figure 1B) and confirmed by NO release results (Figure 1A).

We investigated whether or not these same dendrimers could directly interact with iNOS and COX-2 enzymes. To examine their ability to bind and modulate the activity of these enzymes, computer-assisted molecular docking simulations were performed using the molecular operating environment (MOE) software. $^{20}$ We hypothesized that dendrimers which inhibit iNOS and COX-2 activity should be able to interact and display favorable binding conformations with these enzymes. Figure 2 summarizes the results obtained for the docking of DG1-A and DG1-OH with iNOS. The most favorable binding conformation for each dendrimer is shown.

We investigated several different binding sites during our docking studies and found that, due to its size, the iNOS active site was the one most likely to interact with low generation dendrimers; it is appropriately large, and it has a critical role in enzyme function (Supplemental Figure 3). The lowest energy docking conformations of DG1-A (Figure 2A) and DG1-OH (Figure $2 \mathrm{H}$ ) displayed favorable $\mathrm{VdW}$ interactions, explained by their lack of protrusion through the VdW molecular surface (gray mesh) of iNOS. When the electrostatic (blue-positive; red-negative) and lipophilic (pink-lipophilic; green-hydrophobic) surfaces were compared, DG1-OH was found to exhibit very favorable interactions on all three arms of the dendrimer, especially in the regions of the aromatic rings and terminal $\mathrm{OH}$ groups (Figure $2 \mathrm{I}-\mathrm{J}$ ). These findings suggest that

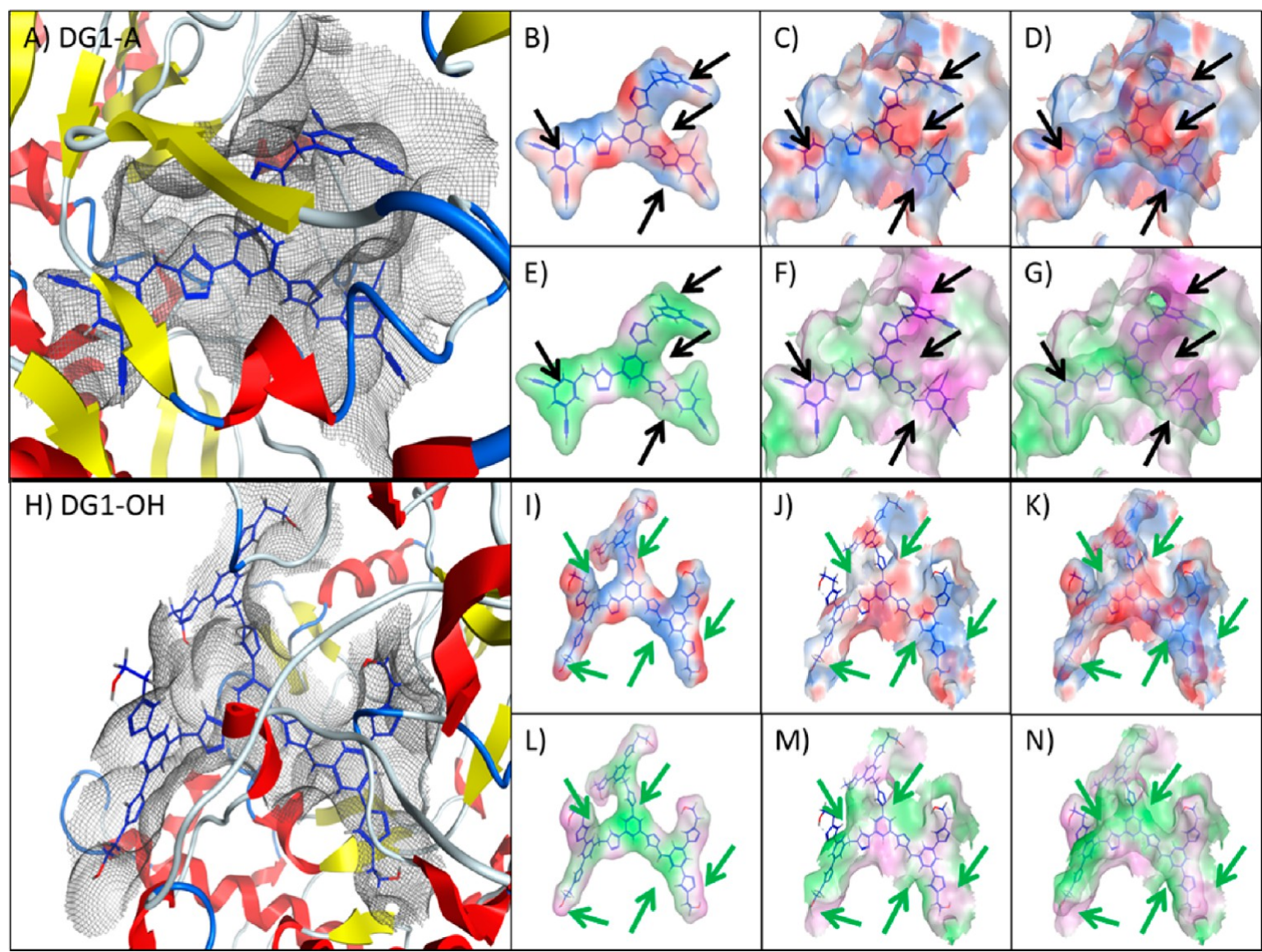

Figure 2. Binding conformation validation for iNOS molecular modeling studies. VdW (A, H), electrostatic (B-D, I-K), and lipophilic (E-G, L$\mathrm{N})$ surface maps for DG1-A and DG1-OH. Regions that are red are considered electronegative, and regions that are blue are considered electropositive. Regions that are green are considered lipophilic, and regions that are purple are considered hydrophilic. 


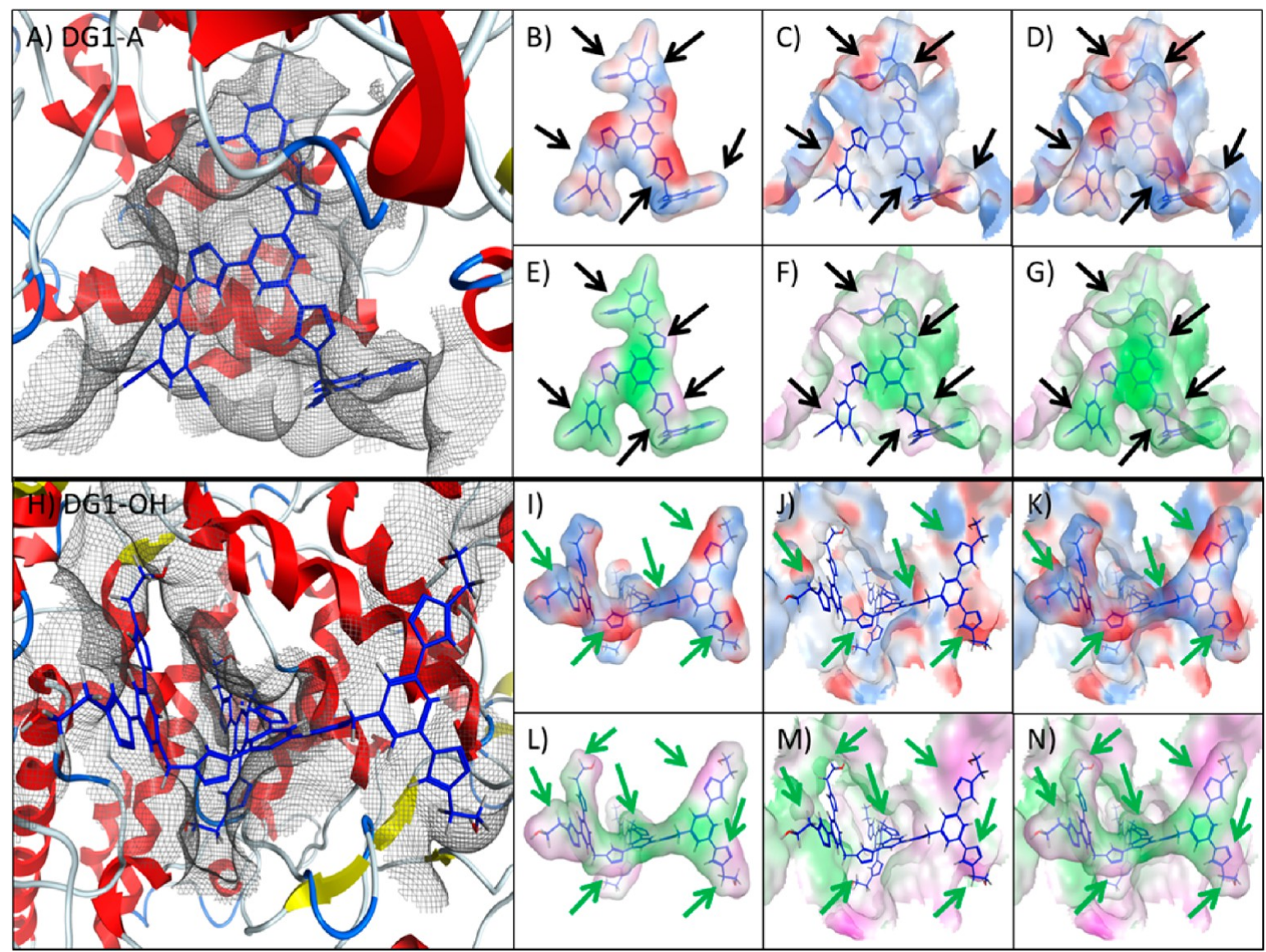

Figure 3. Binding conformation validation for COX-2 molecular modeling studies (Heme site). VdW (A, H), electrostatic (B-D, I-K), and lipophilic $(\mathrm{E}-\mathrm{G}, \mathrm{L}-\mathrm{N})$ surface maps for DG1-A and DG1-OH. Regions that are red are considered electronegative, and regions that are blue are considered electropositive. Regions that are green are considered lipophilic, and regions that are purple are considered hydrophilic.

DG1-OH may bind into the active site of iNOS. However, the best fit docking conformations of DG1-A were not consistent with its interacting successfully with either of the two surfaces of iNOS (Figure 2B-D). DG0-A and DG0-OH displayed overwhelmingly unfavorable interactions with iNOS (Supplemental Figure 4).

In the active site of iNOS, the interaction between Glu377 and the native substrate, L-arginine, is an absolute requirement for normal enzyme function. ${ }^{21}$ Moreover, many well-characterized iNOS inhibitors interact directly with Glu377, obstructing its binding to L-arginine and thus inhibiting iNOS activity. ${ }^{22}$ The ligand interaction map of DG1-OH and iNOS reveals that DG1-OH does not form a hydrogen bond with Glu377 (Supplemental Figure 5). Unlike specific inhibitors of iNOS which bind directly to Glu377, DG1-OH makes other interactions including Met374 and Trp372 which help to stabilize DG1-OH at the active site of iNOS, thereby occluding it. DG1-OH also forms hydrogen bonds with other regions in the active site, most notably Trp463, which contribute to the overall stabilization of the dendrimer in the binding pocket. Findings from our molecular modeling studies suggest that DG1-OH may bind to iNOS (while DG0-OH and DG1-A do not), supporting the likelihood that small dendrimer size influences the anti-inflammatory activity, through interactions with Met374 and Trp463 but not Glu377. These results are in agreement with our biological enzyme activity data (Figure 1). Having identified a likely relationship between the biological results and the molecular modeling data from iNOS, we then performed docking studies using the COX-2 enzyme. In contrast to iNOS, the COX-2 active site is split into two smaller pockets, both of which were predicted to be probable binding sites for the dendrimers. The first site binds the hemoglobin (heme), an essential cofactor, and the second site binds the native ligand, arachidonic acid (AA). Both the heme and the AA sites were investigated individually as potential dendrimer binding pockets.

For DG1-A, there are many prominent sites of unfavorable lipophilicity surface interactions between the triazole rings and the outer benzene rings (Figure 3E-G). In contrast, DG1-OH exhibits not only favorable electrostatics but favorable lipophilicity at the triazole and benzene rings, suggesting that a favorable binding orientation is possible (Figure 3I-K). We therefore concluded that DG1-OH could adopt a favorable binding orientation in the heme site of COX-2, while DG1-A could not. DG0-OH and DG0-A dendrimers showed a similar pattern (Supplemental Figure 6). The highly electronegative and lipophilic DG0-A exhibited unfavorable interactions in at least two arms, while DG0-OH maintained favorable interactions at its triazole rings and terminal hydroxyl groups. Although the AA site is significantly smaller than the heme site, it is conceivable that one arm of the dendrimers might fit and explain the enzyme inhibition. However, molecular docking of the dendrimers into the AA yielded no favorable binding orientations (Supplementary Figures 7 and 8). Taken together, the molecular docking data and biological data of COX-2 suggest that both DG0-OH and DG1-OH can elicit anti- 


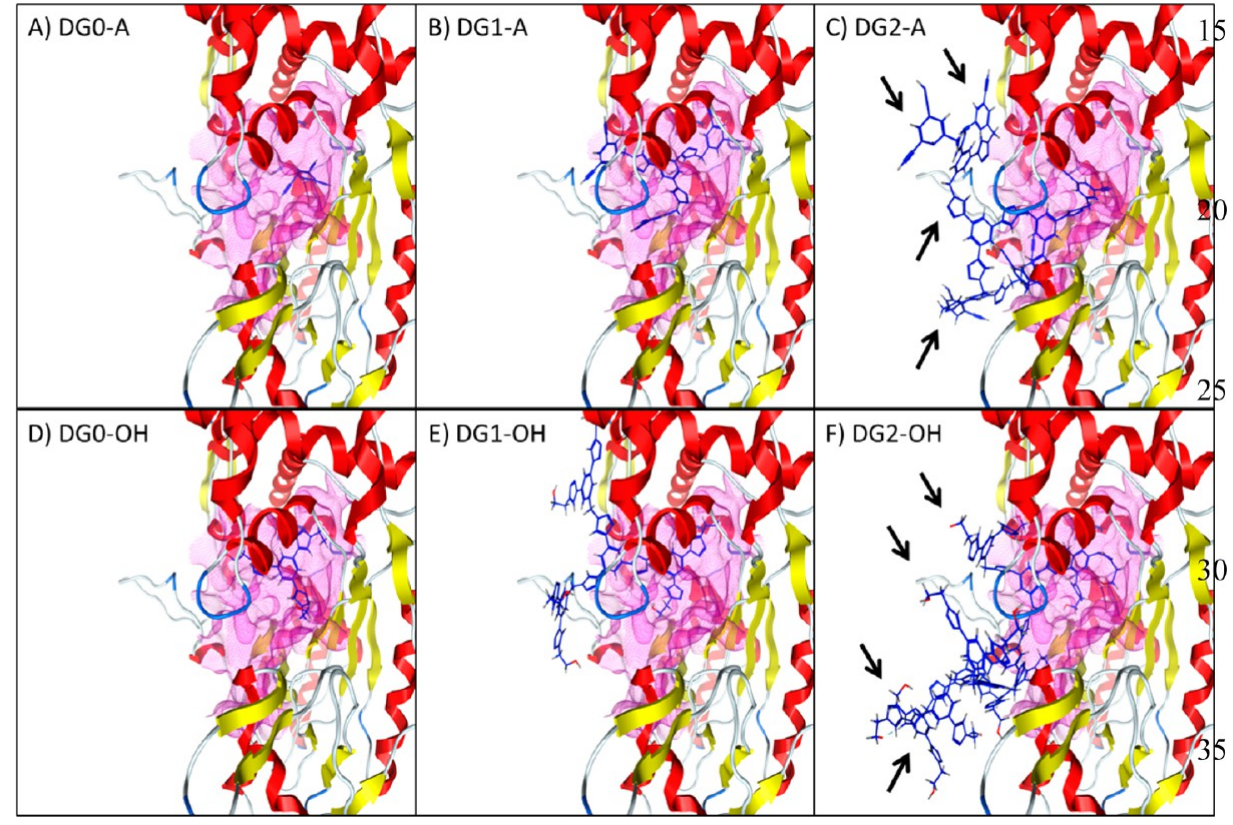

Figure 4. Increased solvent exposure with higher order dendrimers. iNOS secondary structure is represented by red ribbons and yellow sheets. The iNOS active site in shown in pink. Dendrimers are visible in blue. Solvent-exposed regions of docked dendrimers are highlighted by black arrows.

inflammatory activity by binding mainly to the heme site of COX-2. This finding could explain why relatively large concentrations of the dendrimers are required to exert a marked inhibitory effect.

Results from our studies and others show that dendrimers possess anti-inflammatory activity that is related to both dendrimer size and functional groups. ${ }^{12}$ We observed that hydroxyl terminated dendrimers possess greater anti-inflammatory activity than acetylene terminated dendrimers, and first generation dendrimers were more effective than their generation zero counterparts. These increased anti-inflammatory effects are likely due to the electrostatic and lipophilic properties of the dendrimers being more complementary to those of the enzyme binding active site. Molecular orbital calculations show that the anti-inflammatory properties of the dendrimers are reflected in their electrostatic potentials. In general, the highly negative hydroxyl chemical group and weakly positive acetyl terminal groups facilitate binding by establishing nonspecific interactions with the target to occupy the active site, thus inhibiting its activity. In contrast, known specific inhibitors of iNOS and COX-2 are highly conserved and bind to the active site forming key interactions with Glu377 (iNOS) and Arg513 (COX-2), respectively. ${ }^{22,23}$

Our molecular modeling results suggest that the dendrimers of a second generation (G2) and higher are much too large to fit in the active site and would result in highly unfavorable binding interactions (highly solvent exposed) with both iNOS and COX-2 enzymes (Supplemental Figures 9, 10). Our studies suggest that, at most, one arm of the higher generation dendrimer could fit into the active site. As a result, most of the molecule would be solvent-exposed, making the dendrimer/ enzyme interaction energetically unfavorable and highly unstable. A composite image of DG0, DG1, and DG2 dendrimers bound into the iNOS active site is provided in Figure 4 to illustrate this point.

\section{CONCLUSIONS}

In summary, our findings suggest that low generation dendrimers exert varying degrees of anti-inflammatory bioactivity and may directly interact with the active sites of both iNOS and COX-2. Our biological data shows a concentration-dependent inhibition of these enzymes; this is in contrast to the previously observed lack of dose-dependent effect with G4 dendrimers within the low nanomolar concentration range. ${ }^{12}$ Molecular modeling studies suggest that high generation dendrimers interact with iNOS and COX2 differently from low generation ones. DG0 and DG1 dendrimers can adopt favorable binding conformations in the active site of both iNOS and COX-2 enzymes, thereby inhibiting their activity. Conversely, due to the restrictive size of the active site, and increased solvent exposure of the large dendrimer arms, higher generation dendrimers are much less likely to form stable binding interactions. Future studies should employ quantum dynamic analyses and biological investigations with different mutants of iNOS and COX-2 (i.e., Met374 and Trp463 for iNOS) to identify potential interaction sites and provide greater insight into the mechanisms of action of low versus high generation dendrimer as anti-inflammatory agents.

\section{ASSOCIATED CONTENT}

\section{S Supporting Information}

Additional information on the synthesis and characterization of the dendrimers used in this study and a detailed description of the modules and parameters used for the molecular modeling. This material is available free of charge via the Internet at http://pubs.acs.org.

\section{AUTHOR INFORMATION}

\section{Corresponding Author}

*D.M.: Fax: +514-398-6690; tel.: +514-398-1264; e-mail: dusica.maysinger@mcgill.ca. A.K.: Fax: +514-398-3797; tel.: +514-398-6912; e-mail: ashok.kakkar@mcgill.ca. M.A.W.: Tel.: +514-398-6239; e-mail: tony.whitehead@mcgill.ca. 


\section{Author Contributions}

K.N. and V.G. contributed equally.

Notes

The authors declare no competing financial interest.

\section{ACKNOWLEDGMENTS}

We would like to thank Natural Sciences and Engineering Research Council of Canada, (NSERC), Canadian Institutes of Health Research (CIHR), Fonds de Recherche en Sante du Quebec (FRSQ), and Centre for Self-Assembled Chemical Structures (Fonds Quebecois de la Recherche sur la Nature et les Technologies) for financial support. The authors would also like to acknowledge the contributions of Eliza Hutter in the early stages of data collection for the molecular modelling.

\section{REFERENCES}

(1) Fréchet, J. M. J.; Tomalia, D. A. Dendrimers and Other Dendritic Polymers; John Wiley \& Sons, Ltd.: Chichester, 2001.

(2) Tomalia, D. A. Dendritic effects: dependency of dendritic nanoperiodic property patterns on critical nanoscale design parameters (CNDPs). New J. Chem. 2012, 36 (2), 264-281.

(3) Skelly, M. M.; Hawkey, C. J. Potential alternatives to COX 2 inhibitors. BMJ 2002, 324 (7349), 1289-1290.

(4) Dannhardt, G.; Kiefer, W. Cyclooxygenase inhibitors-current status and future prospects. Eur. J. Med. Chem. 2001, 36 (2), 109-126.

(5) Korhonen, R; Lahti, A.; Kankaanranta, H.; Moilanen, E. Nitric oxide production and signaling in inflammation. Curr. Drug Targets Inflamm. Allergy 2005, 4 (4), 471-479.

(6) Fawaz, F.; Bonini, F.; Guyot, M.; Lagueny, A. M.; Fessi, H.; Devissaguet, J. P. Disposition and protective effect against irritation after intravenous and rectal administration of indomethacin loaded nanocapsules to rabbits. Int. J. Pharmaceutics 1996, 133, 107-115.

(7) Gillies, E. R.; Frechet, J. M. Dendrimers and dendritic polymers in drug delivery. Drug Discovery Today 2005, 10 (1), 35-43.

(8) Hayder, M.; Fruchon, S.; Fournie, J. J.; Poupot, M.; Poupot, R. Anti-inflammatory properties of dendrimers per se. Sci. World J. 2011, $11,1367-1382$.

(9) Esfand, R; Tomalia, D. A. Poly(amidoamine) (PAMAM) dendrimers: from biomimicry to drug delivery and biomedical applications. Drug Discovery Today 2001, 6 (8), 427-436.

(10) Weinhart, M.; Groger, D.; Enders, S.; Riese, S. B.; Dernedde, J.; Kainthan, R. K.; Brooks, D. E.; Haag, R. The role of dimension in multivalent binding events: structure-activity relationship of dendritic polyglycerol sulfate binding to L-selectin in correlation with size and surface charge density. Macromol. Biosci. 2011, 11 (8), 1088-1098.

(11) Klajnert, B.; Cortijo-Arellano, M.; Cladera, J.; Bryszewska, M. Influence of dendrimer's structure on its activity against amyloid fibril formation. Biochem. Biophys. Res. Commun. 2006, 345 (1), 21-28.

(12) Chauhan, A. S.; Diwan, P. V.; Jain, N. K.; Tomalia, D. A. Unexpected in vivo anti-inflammatory activity observed for simple, surface functionalized poly(amidoamine) dendrimers. Biomacromolecules 2009, 10 (5), 1195-1202.

(13) Dernedde, J.; Rausch, A.; Weinhart, M.; Enders, S.; Tauber, R.; Licha, K.; Schirner, M.; Zugel, U.; von Bonin, A.; Haag, R. Dendritic polyglycerol sulfates as multivalent inhibitors of inflammation. Proc. Natl. Acad. Sci. U.S.A. 2010, 107 (46), 19679-19684.

(14) Sharma, A.; Khatchadourian, A.; Khanna, K.; Sharma, R.; Kakkar, A.; Maysinger, D. Multivalent niacin nanoconjugates for delivery to cytoplasmic lipid droplets. Biomaterials 2011, 32 (5), 1419-1429.

(15) Castonguay, A.; Wilson, E.; Al-Hajaj, N.; Petitjean, L.; Paoletti, J.; Maysinger, D.; Kakkar, A. Thermosensitive dendrimer formulation for drug delivery at physiologically relevant temperatures. Chem. Commun. (Cambridge) 2011, 47 (44), 12146-12148.

(16) Sharma, A.; Neibert, K.; Sharma, R.; Hourani, R.; Maysinger, D.; Kakkar, A. Facile Construction of Multifunctional Nanocarriers Using
Sequential Click Chemistry for Applications in Biology. Macromolecules 2011, 44 (3), 521-529.

(17) Hourani, R.; Sharma, A.; Kakkar, A. Designing dendritic frameworks using versatile building blocks suitable for CuI-catalyzed alkyne azide "click" chemistry. Tetrahedron Lett. 2010, 51 (29), 37923795.

(18) Stansley, B.; Post, J.; Hensley, K. A comparative review of cell culture systems for the study of microglial biology in Alzheimer's disease. J. Neuroinflamm. 2012, 9, 115.

(19) Oh, Y. T.; Lee, J. Y.; Lee, J.; Lee, J. H.; Kim, J. E.; Ha, J.; Kang, I. Oleamide suppresses lipopolysaccharide-induced expression of iNOS and COX-2 through inhibition of NF-kappaB activation in BV2 murine microglial cells. Neurosci. Lett. 2010, 474 (3), 148-153.

(20) Vilar, S.; Cozza, G.; Moro, S. Medicinal chemistry and the molecular operating environment (MOE): application of QSAR and molecular docking to drug discovery. Curr. Top. Med. Chem. 2008, 8 (18), 1555-1572.

(21) Crane, B. R.; Arvai, A. S.; Gachhui, R.; Wu, C.; Ghosh, D. K.; Getzoff, E. D.; Stuehr, D. J.; Tainer, J. A. The structure of nitric oxide synthase oxygenase domain and inhibitor complexes. Science 1997, 278 (5337), 425-431.

(22) Garcin, E. D.; Arvai, A. S.; Rosenfeld, R. J.; Kroeger, M. D.; Crane, B. R.; Andersson, G.; Andrews, G.; Hamley, P. J.; Mallinder, P. R.; Nicholls, D. J.; St-Gallay, S. A.; Tinker, A. C.; Gensmantel, N. P.; Mete, A.; Cheshire, D. R.; Connolly, S.; Stuehr, D. J.; Aberg, A.; Wallace, A. V.; Tainer, J. A.; Getzoff, E. D. Anchored plasticity opens doors for selective inhibitor design in nitric oxide synthase. Nat. Chem. Biol. 2008, 4 (11), 700-707.

(23) DeWitt, D. L. Cox-2-selective inhibitors: the new super aspirins. Mol. Pharmacol. 1999, 55 (4), 625-631. 